\title{
Tendência Genética para Características Produtivas em Bovinos da Raça Pardo-Suíça
}

\author{
Cláudio Vieira de Araújo1, Robledo de Almeida Torres², Francisco Palma Rennó2 ${ }^{2}$, José Carlos \\ Pereira $^{3}$, Carmen Silva Pereira ${ }^{3}$, Simone Inoe Araújo ${ }^{2}$, Rodolpho de Almeida Torres Filho ${ }^{2}$, \\ Herluce Cavalcanti da Silva ${ }^{4}$, Luciana Navajas Rennó ${ }^{2}$, Fernando da Rocha Kaiser ${ }^{5}$
}

\begin{abstract}
RESUMO - Neste estudo foram analisados registros de produção de leite e de gordura de 4.959 lactações, de 2.414 vacas da raça Pardo-Suiça, filhas de 70 reprodutores, com partos observados de 1985 a 1998, em 51 rebanhos. Foram estimadas as tendências genéticas para as produções de leite (PL305ME) e de gordura (PG305ME), ajustadas à idade adulta e 305 dias de lactação. A tendência genética para os reprodutores foi estimada pela regressão linear dos valores genéticos dos reprodutores, em função do ano de parto de suas filhas, ponderados pelo número de filhas do reprodutor em cada ano de parto. O modelo animal utilizado para estimar os valores genéticos incluiu os efeitos fixos de rebanho, ano-época de parto e grupo genético do animal, além dos efeitos aleatórios, genético aditivo, de ambiente permanente e ambiente temporário. As médias para as produções de leite e gordura foram $6085,79 \pm 1627,73 \mathrm{~kg}$ e $225,60 \pm 60,46 \mathrm{~kg}$, respectivamente. A tendência genética estimada para os reprodutores foi 33,01 $\pm 15,27$ e 1,98 \pm 0,61 kg/ano para PL305ME e PG305ME,

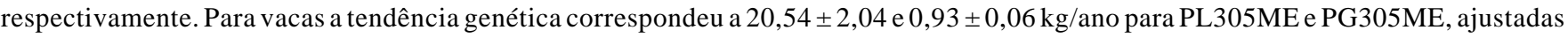
para idade adulta, respectivamente.
\end{abstract}

Palavras-chave: produção de leite, produção de gordura, tendência genética, Pardo-Suíço

\section{Genetic Trend in Productive Traits to Brown Swiss Breed}

\begin{abstract}
Records of 4959 lactation in 1985 to 1998 of 2414 Brown Swiss cows breed, daughters of 70 sires in 51 herds, were used to estimate the genetic trend for milk and fat yield adjusted for 305 days of lactation and mature age of cows (PL305ME and PG305ME). The genetic trend for sires was estimated using a linear regression of the breeding values of the sires to their daughters calving year, averaged to number of daughters in each calving year. The genetic trend of the cows was estimated using a linear regression of their estimates breeding values averaged to number of observation in each year of calving. The animal model used to estimate the breeding values, inclued the fixed effects of herd, year-season of calving, genetic group of the animals, and random effects of animal, permanent and temporary environment. The means of PL305ME and PG305ME were 6085.79 \pm 1627.73 and $225.60 \pm 60.46 \mathrm{~kg}$, respectivelly. The genetic trend estimated to sires were $33.01 \pm 15.27$ and $1.98 \pm 0,61 \mathrm{~kg} /$ year to PL305ME and PG305ME, respectivelly. The genetic trend estimated for cows were $20.54 \pm 2.04$ and $.93 \pm .06 \mathrm{~kg} / \mathrm{year}$ para PL305ME and PG305ME, respectively.
\end{abstract}

Key Words: fat and milk yield, genetic trend, Brown Swiss

\section{Introdução}

O desempenho produtivo de um animalé atribuído a combinação do potencial genético e o ambiente de criação. Para uma população, as alterações nas características produtivas, ao longo dos anos, também têm influência de fatores ambientais e da constituíção genética dos indivíduos. O objetivo principal de um programa de melhoramento genético animal consiste em alterar a média para o caráter desejado. Este objetivo é obtido pela alteração da frequência gênica, na população para os genes que regulam o caráter desejado, ou seja, pela seleção.
Esta mudança de freqüência gênica no carcáter estudado, em direção da fixação de determinados alelos na população, provoca alterações no valor genético médio dos indivíduos ao longo do tempo durante a execução do programa de seleção e a tendência genética pode ser definida como um procedimento estatístico que procura quantificar esta mudança por unidade de tempo. De acordo com Hudson \& Kennedy (1985), a eficácia de um programa de seleção pode ser avaliada por meio da estimação das tendências genéticas na população. Albuquerque et al. (1996) afirmam que, para predizer o ganho genético em determinado programa de seleção, pode-se

\footnotetext{
1 Departamento de Zootecnia- Universidade Federal Rural da Amazônia: araujocv@bol.com.br

2 Doutorando da UFV. E.mail: rodolphotfilho@hotmail.com; fprenno@mail.ufv.br

3 Professor da UFV. E.mail: rtorres@mail.ufv.br

4 Mestre em Zootecnia. E.mail: herluce@bol.com.br

5 Associação Brasileira dos Criadores de Gado Pardo-Suíço.
} 
utilizar a informação contida nos anos de execução deste programa, regredindo a média do valor genéticos em cada ano de nascimento ou parto para obter uma estimativa da tendência genética.

Chenette et al. (1982) relatam que é difícil quantificar as alterações provocadas pelos processos seletivos em uma população. Porém, existem métodos que permitem a identificação de causas responsáveis pelas mudanças na média da população, como resultado de variações no mérito genético e das condições de ambiente.

Estimativas de tendências genéticas são, geralmente, obtidas pela regressão dos valores genéticos dos animais, em função do ano de nascimento ou ano de parto das fêmeas, as duas estimativas refletem respostas diferentes. No primeiro caso, obtém-se uma resposta da contribuição dos reprodutores, quando estes foram utilizados nos rebanhos pela primeira vez, refletindo quais animais estariam contribuindo mais para o programa de seleção. No segundo caso, obtém-se resposta do comportamento do valor genético médio dos animais utilizados a cada ano para gerar nova geração de animais, refletindo como o programa de seleção tem-se comportado nos últimos anos.

Estudando dados de 34.380 primeiras lactações em 355 rebanhos da raça Holandesa nos Estados Unidos, Burnside \& Legates (1967) encontraram estimativas de tendência genética anuais de 45 a $55 \mathrm{~kg}$. Resultado inferior foi encontrado por Verde et al. (1972), que obtiveram estimativa de $33 \mathrm{~kg} / \mathrm{ano}$ para a produção de leite em rebanhos da raça Holandesa, na Florida. Já Olson \& Jensen (1976), nos Estados Unidos, encontraram estimativa de tendência genética para a produção de leite de $79 \mathrm{~kg}$, com desvio-padrão de $25,7 \mathrm{~kg} / \mathrm{ano}$ para a raça Holandesa.

Utilizando-se registros de produção de leite e de gordura de animais oriundos de diferentes raças, Everett et al. (1976) estimaram a tendência genética para rebanhos leiteiros nos Estados Unidos. As estimativas de tendências genéticas para os animais das raças Ayrshire, Guernsey, Holandês, Jersey e PardoSuíça foram de $17,7,23,11$ e $23 \mathrm{~kg} /$ ano para a produção de leite e de $0,7,0,3,0,5,0,1$ e $0,8 \mathrm{~kg} / \mathrm{ano}$ para a produção de gordura.

Musani \& Mayer (1997), por meio de registros de lactações de vacas Jersey, estimaram a tendência genética para a produção de leite em $0,8 \mathrm{~kg} / \mathrm{ano}$. Concluíram que, em decorrência da alta variação genética para a produção, a tendência genética foi baixa, refletindo a ineficiência da seleção. No entanto, a tendência ambiental indicou substancial progresso no manejo.

Tendências genéticas para as produções de leite e gordura, em animais da raça Holandesa, foram estimadas por Durães et al. (2001) no Brasil, por meio de 18.482 registros separados em dois períodos de tempo. Os valores estimados de tendência genética foram 18,4 e 0,6 kg/ano para produção de leite e gordura, respectivamente. Os autores concluíram que os resultados foram menores em relação aos teoricamente possíveis de serem alcançados, apesar da constatação de aumento das médias de produções no decorrer dos anos, provavelmente em decorrência de melhorias de manejo e nutrição dos animais.

Verneque et al. (1996), utilizando registros de produção de leite em animais Gir, encontraram estimativa de tendência genética igual a 13,88 $\pm 0,92 \mathrm{~kg}$ / ano, concluíndo que o incentivo e ampliação de programas de teste de progênie poderiam resultar em maior disponibilidade de touros provados, o que resultaria em melhores opções para incremento do potencial genético da raça no Brasil. Também em animais da raça Gir, Ledic (1992) encontrou tendência genética igual a $2,27 \mathrm{~kg} /$ ano de leite.

Estudando dados de produção de leite em rebanhos da raça Holandesa nos Estados Unidos, Powell \& Wiggans (1991) verificaram ganho correspondente a $87 \mathrm{~kg} /$ ano de leite sobre a média de produção por ano. Van Tassel \& Van Vleck (1991) afirmaram que, devido a baixos diferenciais de seleção e altos intervalos de gerações, o ganho genético anual observado foi abaixo da expectativa, para os rebanhos americanos especializados na atividade leiteira. Ganhos genéticos anuais iguais a 24 e $33 \mathrm{~kg}$ para produção de leite e de 0,64 e $0,85 \mathrm{~kg}$ para a produção de gordura foram obtidos por Costa (1999), em animais da raça Holandesa, no Brasil.

Silva et al. (2000) verificaram, para animais do ecótipo Mantiqueira no Brasil, ganhos genéticos anuais negativos de $-14,27$ e - $-0,10$, e de 3,36 e 0,10 paras as produções de leite e gordura, para os reprodutores e vacas, respectivamente.

O objetivo do presente estudo foi estimar a tendência genética para a produção de leite e gordura em rebanhos da raça Pardo-Suíça no Brasil.

\section{Material e Métodos}

Foram utilizados registros de 4.959 lactações referentes às produções de leite e gordura de 2.414 
vacas da raça Pardo-Suíça, filhas de 70 reprodutores, distribuídos em 51 rebanhos. Os registros são provenientes do serviço de controle leiteiro da Associação Brasileira de Criadores de Gado Pardo Suíço, com os partos ocorridos entre os anos de 1985 e 1998. Os registros de produções de leite e de gordura foram agrupados em quatros épocas, segundo o mês de parto: janeiro a março, abril a junho, julho a setembro e outubro a dezembro. Com referência à informação sobre ascendência e origem das vacas, os animais foram classificados em Grupo 1, animais puro de origem, Grupo 2, animais puros por cruza; e Grupo 3, animais importados, puros de origem.

As produções de leite e gordura foram ajustadas para duas ordenhas, 305 dias de lactação e para a idade adulta da vaca $(77,5$ meses para a produção de leite e 65,5 meses para a produção de gordura), mediante o uso de fatores estimados por Rennó (2001).

Para a análise, foi utilizado o método da máxima verossimilhança restrita e um modelo animal considerando os efeitos fixos de rebanho, ano-estação e grupo genético do animal e os efeitos aleatórios de animal, de ambiente permanente e ambiente temporário, descrito matricialmente como:

$$
\mathrm{y}=\mathrm{X} \underset{\sim}{\mathrm{b}}+\mathrm{Z}_{\mathrm{a}} \underset{\sim}{\mathrm{a}}+\mathrm{Z}_{\mathrm{p}} \underset{\sim}{\mathrm{p}}+\underset{\sim}{\mathrm{e}}
$$

em que $\mathrm{y}$ é um vetor $\mathrm{n}$ x 1 , de $\mathrm{n}$ observações referentes as produções de leite e gordura, $\mathrm{X}$ é a matriz de incidência dos efeitos fixos de rebanho, ano-época de parto e grupo genético do animal, $\mathrm{Z}_{\mathrm{a}} \mathrm{e}$ $Z_{p}$ são as matrizes de incidência dos efeitos aleatórios genético-aditivo e de ambiente permanente de animal, associados aos vetores $\underset{\sim}{\mathrm{a}}$ e $\underset{\sim}{\mathrm{p}}$, dos valores genéticos aditivos e permanente de ambiente, respectivamente, e ainda, $\underset{\sim}{\mathrm{e}}$ é o vetor de resíduos de mesma dimensão de $\mathrm{y}$.

As pressuposições acerca da distribuição dos vetores $\mathrm{y}, \underset{\sim}{\mathrm{b}}, \underset{\sim}{\mathrm{a}}, \mathrm{p}$ e e podem ser descritas como:

$$
\left[\begin{array}{c}
\mathrm{y} \\
\tilde{\mathrm{a}} \\
\tilde{\mathrm{p}} \\
\tilde{\mathrm{e}}
\end{array}\right] \cong\left\{\left[\begin{array}{c}
\mathrm{Xb} \\
\phi \\
\phi \\
\phi
\end{array}\right],\left[\begin{array}{cccc}
\mathrm{Z}_{\mathrm{a}} \mathrm{GZ}_{\mathrm{a}}^{\prime}+\mathrm{Z}_{\mathrm{p}} \mathrm{PZ}_{\mathrm{p}}^{\prime}+\mathrm{R} & \mathrm{Z}_{\mathrm{a}} \mathrm{G} & \mathrm{Z}_{\mathrm{p}} \mathrm{P} & \mathrm{R} \\
\mathrm{GZ}_{\mathrm{a}}^{\prime} & \mathrm{G} & \phi & \phi \\
\mathrm{PZ}_{\mathrm{p}}^{\prime} & \phi & \mathrm{P} & \phi \\
\mathrm{R} & \phi & \phi & \mathrm{R}
\end{array}\right]\right\},
$$

em que $\mathrm{G}=\mathrm{A} \otimes \mathrm{G}_{0}, \mathrm{P}=\mathrm{I}_{\mathrm{N}} \otimes \mathrm{P}_{0}$ e $\mathrm{R}=\mathrm{I}_{\mathrm{n}} \otimes \mathrm{R}_{0}$, sendo A uma matriz cujo os elemento são os numeradores do coeficiente de parentesco entre os ani- mais, de ordem igual ao número de indivíduos $(\mathrm{N}) ; \mathrm{G}_{0}$ é a matriz de (co)variância genética aditiva entre as características; $\mathrm{I}_{\mathrm{N}}$ é uma matriz identidade, de ordem $\mathrm{N} ; \mathrm{P}_{0}$ é a matriz de variância referente ao efeito de ambiente permanente sobre o animal, $\mathrm{I}_{\mathrm{n}}$ matriz identidade, de ordem igual ao número total de observações (n); e $\mathrm{R}_{0}$ é matriz de variância residual entre as características.

As equações do modelo misto para as produções de leite e gordura são descritas como:

$$
\left[\begin{array}{ccc}
X^{\prime} R^{-1} X & X^{\prime} R^{-1} Z & X^{\prime} R^{-1} Z \\
Z^{\prime} R^{-1} X & Z^{\prime} R^{-1} Z+G^{-1} & Z^{\prime} R^{-1} Z \\
Z^{\prime} R^{-1} X & Z^{\prime} R^{-1} Z & Z^{\prime} R^{-1} Z+P^{-1}
\end{array}\right]\left[\begin{array}{c}
\beta^{0} \\
\tilde{\hat{a}} \\
\tilde{\hat{p}}
\end{array}\right]=\left[\begin{array}{c}
X^{\prime} R^{-1} \tilde{y} \\
Z^{\prime} R^{-1} \tilde{y} \\
Z^{\prime} R^{-1} \tilde{y}
\end{array}\right]
$$

Os parâmetros genéticos utilizados foram os mesmos obtidos por Rennó (2001), sendo as herdabilidades assumidas para as produções de leite e de gordura iguais a 0,37 e 0,36 , respectivamente. A correlação genética entre as duas características foi igual a 0,96 e a repetibilidade para as produções de leite e gordura foram iguais a 0,40 e 0,37 , respectivamente.

A tendência genética para os reprodutores foi estimada pela regressão linear simples dos valores genéticos dos reprodutores, em função do ano de parto em que os reprodutores tiveram suas primeiras filhas, ponderado pelo número de filhas do reprodutor em cada ano de parto. Para estimar a tendência genética das vacas, ajustou-se um modelo de regressão linear simples da média dos valores genéticos, em função do ano de parto das vacas, usando como fator de ponderação o número de observações em cada ano de parto.

As soluções de equações do modelo misto foram obtidas por meio do programa MTDFREML (Boldman et al., 1995).

\section{Resultados e Discussão}

As médias estimadas e respectivos desvios-padrão das produções de leite e de gordura foram iguais a $6.085,79 \pm 1.629,73 \mathrm{~kg}$ e $225,61 \pm 60,47 \mathrm{~kg}$, respectivamente. A estimativa de tendência genética para os reprodutores foi 33,01 $\pm 15,27$ e 1,98 $\pm 0,61 \mathrm{~kg} / \mathrm{ano}$ para as produções de leite e gordura, respectivamente. Para as vacas, a tendência genética correspondeu a $20,54 \pm 2,04$ e $0,93 \pm 0,06 \mathrm{~kg} /$ ano para as produções de leite e gordura, respectivamente. As tendências genéticas para as produções de leite e gordura, embora positivas (Figuras 1 e 2), são de baixa mag-

R. Bras. Zootec., v.32, n.6, p.1872-1877, 2003 (Supl. 2) 


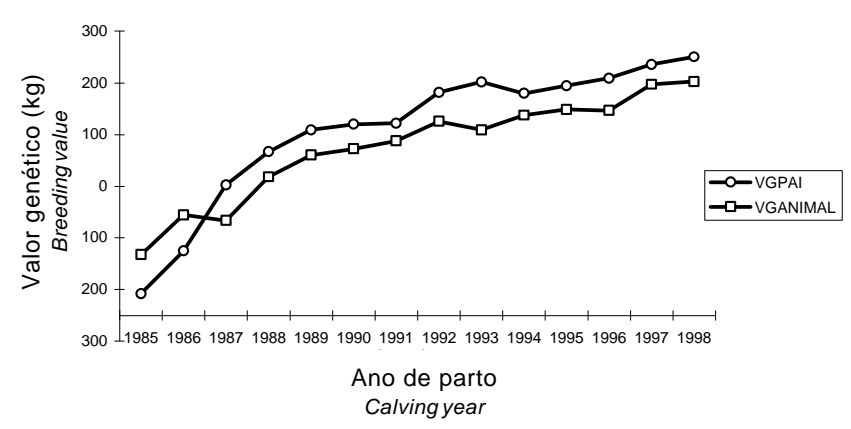

Figura 1 - Estimativas dos valores genéticos médios dos reprodutores (VGPAI) e das vacas (VGANIMAL) para a produção de leite em função do ano de parto.

Figure 1 - Means of predicted breeding value estimates for sires (VGPAI) and cows (VGANIMAL) for milk yield by calving year.

nitude, correspondendo a 0,55 e $0,88 \%$ das médias das produções de leite e de gordura, respectivamente. Os valores são menores que os $2 \%$ teoricamente possíveis de serem alcançados, conforme relatado por Kim et al. (1999). Durães et al. (2001) discutem que possíveis causas para os baixos valores de tendência genética anuais no Brasil, relatados na literatura, poderiam ser reflexo de falhas na escolha de reprodutores, utilização de touros com baixa acurácia, e portanto de menor preço, com o objetivo de redução de custos.

Wattiaux (1995) afirma que novas metodologias de avaliação genética permitem aumentar a precisão destas avaliações, contribuindo para o aumento da produção média nos rebanhos. Em um contexto geral, resultados de tendências genéticas encontradas no Brasil para as produções de leite e gordura têm sido menores que o possível de ser obtido (Verneque et al., 1996; Costa et al., 1999; Durães et al., 2001; Silva et al., 2000).

As tendências genéticas para as produção de leite e de gordura, neste estudo, apresentaram comportamento similar e demonstram ganhos genéticos maiores nos últimos anos avaliados (Figuras 1 e 2). Este resultado pode ser reflexo da utilização de sêmen de reprodutores com maiores capacidades previstas de transmissão, produzindo progênies com maior potencial genético. Além disso, devido à maior intensidade de seleção ser praticada nos reprodutores, estes poderiam estar contribuindo com a maior parcela das médias dos valores genéticos preditos para ambas as características em cada ano de parto, e conseqüentemente, imprimindo em anos de parto

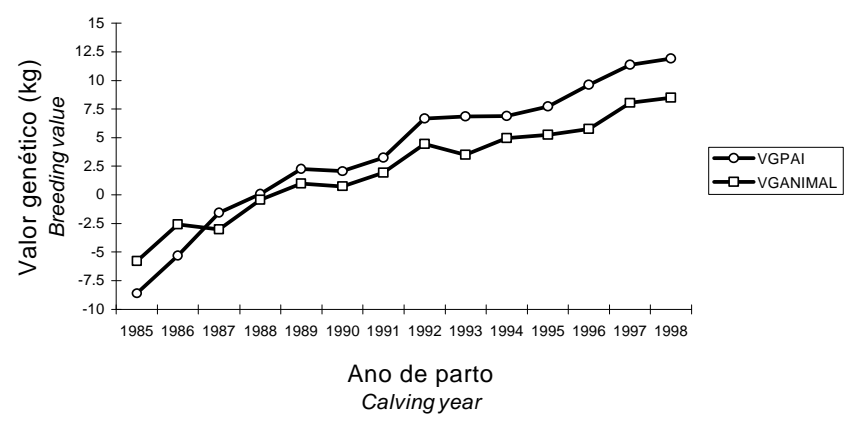

Figura 2 - Estimativas dos valores genéticos médios dos reprodutores (VGPAI) e das vacas (VGANIMAL) para a produção de gordura em função do ano de parto.

Figure 2 - Means of predicted breeding value estimates for sires (VGPAl) and cows (VGANIMAL) for fat yield by calving year.

posteriores maior potencial produtivos em suas filhas.

As médias fenotípicas para as produções de leite e de gordura aumentaram com o decorrer do período avaliado (Tabela 1). As produções médias de leite variaram de 1985 a 1998 , de $4.172,54 \mathrm{~kg}$ a $7.222,93 \mathrm{~kg}$, demonstrando um aumento na produção de leite, no período avaliado de $3.050,39 \mathrm{~kg}$, ou seja, $217,9 \mathrm{~kg}$ de leite, em média, por ano. Para a produção de gordura, o aumento no período avaliado foi de $108,11 \mathrm{~kg}$, correspondendo a $7,72 \mathrm{~kg}$ de gordura, em média, por ano. Confrontando os valores das tendências genéticas estimadas para as produções de leite e de gordura com as médias fenotípicas estimadas em cada ano de parto avaliado, infere-se que os maiores valores para as produções médias nos últimos anos refletem, provavelmente, além do efeito favorável da seleção genética, o melhoramento de fatores não genéticos.

A seleção para animais de alta produção, demanda mudanças no manejo nutricional dos rebanhos, uma vez que animais mais produtivos apresentam maior capacidade de ingestão de matéria seca, bem como maiores exigências nutricionais. É fato que, quando os animais são selecionados geneticamente para a produção de leite, é a sua produção que determina a quantidade de nutrientes que deve ingerir (Andriguetto, 1983). Em rebanhos cujos animais não estejam recebendo os nutrientes necessários para atendimento de suas necessidades nutricionais, o animal irá mobilizar os nutrientes recebidos pela dieta para a sua função genética principal, produção de leite, mesmo a custa de sua mantença. No caso da energia para a produção de leite, o animal irá degradar suas reservas lípidicas, quando a alimentação é 
insuficiente para atender suas necessidades para a produção, o que, juntamente com deficiência de fósforo, característica das pastagens tropicais, compromete a síntese de hormônios esteróides, afetando a reprodução e, conseqüentemente, a próxima lactação, aumentando o intervalo de partos, que por sua vez, provoca valores nulos de tendência genética.

Outra possível explicação para baixos valores de estimativas de tendência genética é o fluxo desorganizado do melhoramento genético, onde rebanhos de mérito genético inferior, fornecem reprodutores para rebanhos de maior mérito genético, fazendo com que o efeito da seleção para maiores produções se cancele. Isto se deve a metodologias inadequadas para a avaliação genética dos animais, ocasionando na escolha inadequada dos animais destinados à reprodução.

Aumento na média da produção de gordura nos anos mais recentes, além do mérito genético dos animais, pode ser reflexo também do manejo das pastagens, uma vez que a síntese de gordura de leite em ruminantes é feita a partir do ácido acético, e a quantidade de ácido acético produzida pelo rúmen está relacionada com o consumo de carboídratos estruturais, principalmente celulose e hemicelulose. Pastagens muito tenras apresentam menor teor de fibra bruta, ao passo que pastagens com avançado

Tabela 1 - Médias e desvios-padrão para as produções de leite e gordura, em $\mathrm{kg}$, e o número de observações em cada ano de parto

Table 1 - Means and standard errors for milk and fat yields $(\mathrm{kg})$ and number of observations $(N)$ in each calving year

\begin{tabular}{lccccc}
\hline & & $\begin{array}{c}\text { Produção } \\
\text { de leite }(\mathrm{kg}) \\
\text { Milkyield }\end{array}$ & & & $\begin{array}{c}\text { Produção de } \\
\text { gordura (kg) } \\
\text { Fatyield }\end{array}$ \\
Ano (Year) & $\mathrm{N}$ & $\overline{\mathrm{X}} \mathrm{EP}$ & & $\overline{\mathrm{X}} \mathrm{EP}$ \\
\hline 1985 & 147 & $4172,54 \pm 1190,69$ & & $164,18 \pm 46,11$ \\
1986 & 151 & $4544,18 \pm 1138,87$ & & $176,95 \pm 43,82$ \\
1987 & 127 & $4993,09 \pm 993,34$ & & $197,39 \pm 38,09$ \\
1988 & 205 & $5276,57 \pm 1242,24$ & & $200,14 \pm 47,06$ \\
1989 & 283 & $5409,81 \pm 1235,89$ & & $204,36 \pm 50,24$ \\
1990 & 306 & $5330,32 \pm 1251,42$ & & $200,64 \pm 48,69$ \\
1991 & 349 & $5578,43 \pm 1279,09$ & & $205,49 \pm 47,14$ \\
1992 & 413 & $5746,44 \pm 1291,59$ & & $206,47 \pm 45,69$ \\
1993 & 517 & $6057,84 \pm 1524,58$ & & $216,49 \pm 49,81$ \\
1994 & 524 & $6292,15 \pm 1545,12$ & & $232,36 \pm 55,14$ \\
1995 & 652 & $6657,18 \pm 1642,78$ & & $243,27 \pm 58,86$ \\
1996 & 590 & $6752,42 \pm 1535,41$ & & $251,38 \pm 62,69$ \\
1997 & 309 & $6926,90 \pm 1586,79$ & & $258,59 \pm 61,18$ \\
1998 & 386 & $7222,93 \pm 1574,25$ & & $272,29 \pm 65,17$ \\
\hline
\end{tabular}

R. Bras. Zootec., v.32, n.6, p.1872-1877, 2003 (Supl. 2) ciclo vegetativo apresentam fibras de menor digestão pelos microorganismos do rúmen.

Nas últimas décadas, o ganho genético para as produções de leite e de gordura tem sido bastante substancial, nos países que adotam práticas adequadas de criação. A evolução de metodologias de avaliação genética apoiadas na evolução da microinformática, bem como o uso popularizado de modernas técnicas de reprodução, tem dado suporte a consideráveis mudanças nos ganhos genético nestes rebanhos.

O aumento da produção dos rebanhos brasileiros é um objetivo cujo alcance demanda de esforço consistente por parte de pesquisas nas áreas relacionadas à produção animal. A seleção e uso de animais provados geneticamente superiores tem conduzido, ainda que de forma lenta, a mudanças significativas nas médias das características importantes economicamente.

Os resultados revelam que, provavelmente, houve melhoramento no manejo dos animais, que em conjunto com o progresso genético dos rebanhos, nos últimos anos avaliados (Figuras 1 e 2), provocaram acréscimo nas produções médias.

\section{Conclusões}

Nos rebanhos da raça Pardo-Suíça estudados, os produtores têm conseguido promover melhoria genética nas produções de leite e gordura, sendo que este progresso genético foi maior nos anos mais recentes. No entanto, em razão dos baixos valores para as tendências genéticas, é possível afirmar que os aumentos médios nas produções de leite e gordura são reflexos de melhorias nos fatores não genéticos, como manejo e alimentação dos animais.

Contudo, os resultados sugerem a possibilidade de se atingirem maiores progressos genéticos, visto que há grande variabilidade genética para as produções de leite e de gordura nos animais da raça Pardo-Suíça.

Maiores ganhos genéticos anuais podem ser alcançados para a raça Pardo-Suiça no Brasil, dando-se maior ênfase à utilização de reprodutores provados em programa de avaliação genética nacional. Com a identificação dos melhores reprodutores e reprodutrizes, capazes de disseminar, em um fluxo organizado, alelos responsáveis pelo incremento das produções de leite e gordura, torna-se possível alterar positivamente as frequiências gênicas da população brasileira da raça Pardo-Suíço. 


\section{Literatura Citada}

ANDRIGUETTO, J.M., PERLY, L.; MINARDI, I. et al. Nutrição animal. 3.ed. São Paulo: Nobel, 1983. v.1-2, 256p.

ALBUQUERQUE, L.G.; QUEIROZ, S.A.; DIAS, A.S.C. et al. Comparação e questionamentos sobre metodologias para estimar tendências genéticas. In: REUNIÃO DA SOCIEDADE BRASILEIRA DE ZOOTECNIA, 33., 1996, Fortaleza. Anais... Fortaleza: Sociedade Brasileira de Zootecnia, 1996. p.313-317.

BOLDMAN, K.G.; KRIESE, L.A.; Van VLECK, L.D. et al. A manual for use of MTDFREML: a set of programs to obtain estimates of variances and covariances (DRAFT). Lincoln: Department of Agricuture/Agriculture Research Service, 1995. 125p.

BURNSIDE, E.B.; LEGATES, J.E. Estimation of genetic trends in dairy cattle populations. Journal of Dairy Science, v.50, n.9, p.1448-1457, 1967.

CHENETTE, C.G.; FRAHM, R.R.; WHITEMAN, J.V. Direct and correlated responses to selectionfor increase wearningand yearling weights in Hereford cattle. II. Evaluation of response. Animal Science Research Reproduction, v.112, p.301-307, 1982.

COSTA, C.N.; BLAKE, R.W.; POLLAK, E.J. et al. Tendências genéticas das produções de leite e gordura na raça Holandesa no Brasil. In: REUNIÃO DA SOCIEDADE BRASILEIRA DE ZOOTECNIA, 36., 1999, Porto Alegre. Anais... Porto Alegre: Sociedade Brasileira de Zootecnia, 1999. p.131.

DURÃES, M.C.; FREITAS, A.F.; VALENTE, J. et al. Tendência genética para a produção de leite e de gordura em rebanhos da raça Holandesa no estado de Minas Gerais. Revista Brasileira de Zootecnia, v.30, n.1, p.66-70, 2001.

EVERETT, R.W.; KEOWN, J.F.; CLAPP, E.E. Production and stayability trends in dairy cattle. Journal of Dairy Science, v.59, n.8, p.1532-1539, 1976.

HUDSON, G.F.S.; KENNEDY, B.W. Genetic evaluation of swine for growth rate and back fat thickness. Journal of Animal Science, v.61, n.1, p.83-91, 1985.

KIM, J.S.; PARK, K.D.; JEONG, H.Y. Estimation of regional genetic trends for milk and fat yields in the Korean Holstein population. Korean Journal of Animal Science, v.41, n.1, p.11-14, 1999.

LEDIC, I.L. Investigação sobre a produção de leite e peso ao parto em gado Gir. Revista da Sociedade Brasileira de Zootecnia, v.21, n.5, p.815-827, 1992.
MUSANI, S.K.; MAYER, M. Genetic and environmental trends in a large commercial Jersey herd in the Central Rift Valley, Kenya. Tropical Animal Health and Production, v.29, n.2, p.108-116, 1997.

OLSON, K.E.; JENSEN, E.L. Estimation of genetic trend in Wisconsin Holsteins. In: ANNUAL MEETING OF THE AMERICAN DAIRY SCIENCE ASSOCIATION, 71., 1976, Raleigth. Proceedings... Raleigth: North Caroline State University, 1976.

POWELL, R.L.; WIGGANS, G.R. Animal model evaluations for Mexican Holsteins. Journal of Dairy Science, v.74, n.4, p.1420-1427,1991.

RENNÓ, F.P. Aspectos produtivos da raça Pardo-Suíça no Brasil. Viçosa, MG: Univeresidade Federal de Viçosa, 2001. 104p. Dissertação (Mestrado em Zootecnia) - Universidade Federal de Viçosa, 2001.

SILVA, M.V.G.B.; GUARAGNA, G.P.; FERREIRA, W.J. et al. Tendência genética sobre os 20 anos de seleção do ecótipo Mantiqueira. 1. Características produtivas. In: REUNIÃO ANUAL DA SOCIEDADE BRASILEIRA DE ZOOTECNIA, 37., 2000, Viçosa, MG. Anais... Viçosa: Sociedade Brasileira de Zootecnia, 2000. p.249.

Van TASSEL, R.L.; Van VLECK, L. D. Estimates of genetic selection differentials and generations interval for four paths of selection. Jounal of Dairy Science, v.74, n.3, p.10781086, 1991.

VERDE, O.G.; WILCOX, C.J.; MARTIN, F.G. et al. Genetic trends in milk production in Florida Dairy Herd improvement Association Herds. Journal of Dairy Science, v.55, n.7, p.1010-1012, 1972.

VERNEQUE, R.S.; FERREIRA, W.J.; TEODORO, R.L. et al. MARTINEZ, M. L.Tendência genética da produção de leite em rebanhos da raça Gir Leiteiro In: REUNIÃO DA SOCIEDADE BRASILEIRA DE ZOOTECNIA, 33., 1996, Fortaleza. Anais... Fortaleza: Sociedade Brasileira de Zootecnia, 1996. p.30-32.

WATTIAUX, M.A. Reproduction and genetic selection.. Madison: University of Wisconsin, 1995. p.111-145 (TECHNICAL DAIRY GUIDE)

Recebido em: $28 / 10 / 01$ Aceito em: $15 / 04 / 03$ 